\title{
Predominance of Plasmodium malariae-falciparum Co-Infection by Molecular Speciation in Bangolan, North West Region of Cameroon
}

\author{
Olivia Afa Achonduh, Aristid Herve Ekollo Mbange, Atogho-Tiedeu Barbara, Innocent Ali Mbulli, Daniel \\ Achinko, Palmer Masumbe Netongo and Wilfred Fon Mbacham \\ The Biotechnology Center, University of Yaounde I, Yaounde, Center Region, Cameroon
}

Received: December 19, 2012 / Accepted: April 12, 2013 / Published: June 30, 2013.

\begin{abstract}
Although microscopy still remains the gold standard for the diagnosis of malaria, rapid diagnostic tests (RDTs) and PCR assays have been shown to be sensitive and specific. Very few comparative studies have been reported of the three diagnostic methods on the same samples in vulnerable groups. Microscopy, RDTs and PCR assays were used for detection and speciation of Plasmodium falciparum (Pf), Plasmodium malariae $(\mathrm{Pm})$ and Plasmodium ovale $(\mathrm{Po})$ in patients in a rice culture savanna ecotype. Fifty four children and 16 pregnant women presenting with a fever were recruited. Bloods collected was used for thin and thick smears, perform RDTs and spotted blood on filter paper for DNA extraction and performance of a PCR. Mean parasitaemia was $37,619.06( \pm 33,599.04) \mathrm{p} / \mu \mathrm{L}$ and 7,512.5 $( \pm 12,446.11) \mathrm{p} / \mu \mathrm{L}$ for children and pregnant women, respectively. A total of $87.14 \%$ were positive by microscopy, $85.71 \%$ by RDTs and $90 \%$ by PCR. Distribution of Plasmodium species as identified by PCR was $72.86 \%$ $\mathrm{Pf} / \mathrm{Pm}, 11.43 \% \mathrm{Pf} / \mathrm{Pm} / \mathrm{Po}$ and $5.43 \% \mathrm{Pm}$ while $10 \%$ were negative. Cohen's Kappa value for PCR and RDTs was K = $0.75(\mathrm{CI}=$ $0.28-1.22)$ while $\mathrm{PCR}$ and microscopy was $\mathrm{K}=0.64(\mathrm{CI}=0.18-1.10)$. Malaria infection in Bangolan was mostly due to mix infection predominantly P. falciparum/P. malariae.
\end{abstract}

Key words: Plasmodium sp., microscopy, rapid diagnostic tests, PCR, children, pregnant women.

\section{Introduction}

Malaria affects 300-500 million people annually and accounts for over 1 million deaths, mainly in African children aged less than five years [1]. It has been estimated that the economic burden of malaria is extremely high, accounting for a reduction of $1.3 \%$ in the annual economic growth rate of countries in which malaria is endemic, and the consequent long-term impact is a reduction of gross national product (GNP) of more than half [2]. Apart from children less than five, pregnant women are potential reservoir for malaria transmission as most of them are asymptomatic in endemic areas. Clinical consequences of placental malaria comprise maternal

Corresponding author: Wilfred Fon Mbacham, Sc.D., associate professor, research fields: public health biotechnology and biochemistry. E-mail: wfmbacham@prd-college.eu. anemia, low birth weight (LBW), preterm delivery and consequently increased prenatal mortality (PM). In sub-Saharan Africa, malaria during pregnancy is estimated to account for $3-8 \%$ of $\mathrm{PM}, \sim 35 \%$ of LBW [3].

Effective treatment of malaria requires precise laboratory diagnosis and this remains a cornerstone for global malaria control efforts. P. falciparum, which can be fatal, must be identified promptly and differentiated from the other Plasmodium species that cause malaria. Microscopy still remains the method of choice in the diagnosis of malaria in endemic areas because it is cost effective. However correct identification of Plasmodium species by microscopy depends on factors such as; the experience of the microscopists, proper staining of the slides, appropriate maintenance of the 
microscope and the time spent reading a slide. The sensitivity of microscopy is approximately 10-30 parasites $/ \mu \mathrm{L}$ of blood [4] and this is usually a challenge in malaria endemic areas. These factors may result in incorrect speciation and in ability to detect mix infections and low parasitaemia. On the other hand, rapid diagnostic tests (RDTs) have been reported to have a considerable potential as a tool to improve the diagnosis of malaria $[5,6]$ and several commercially available tests are sensitive, specific, and stable under operational conditions [7]. However, RDTs have some limitations e.g. HRP-2-based immune- chromatographic tests permit rapid diagnosis of $P$. falciparum malaria, hence their clinical usefulness for the diagnosis of other Plasmodium spp. and for monitoring of the therapeutic response is limited. Since HPR-2 is expressed only by $P$. falciparum, these tests will give negative results with samples containing only $P$. vivax, P. ovale, P. malaria and P. knowlesi. Many cases of non-falciparum malaria may therefore be misdiagnosed as malaria negative. Polymerase chain reaction (PCR) based techniques have been reported to be more sensitive and specific $[8,9]$ and have the ability to detect malaria parasites in patients with low levels of parasitemia and identify them to the species level. It is an attractive addition for confirmation of results of other methods.

Microscopy still remains the gold standard for parasitological diagnosis of malaria in Cameroon despite its disadvantages of electrical supply, well trained technicians, and available reagents amongst others. The government is yet to introduce a policy for the use of RDTs for routine diagnose of malaria and PCR is still restricted only to a few research laboratories. The objective of this study was therefore to compare the specificity and sensitivity of microscopy and the RDTs (SD Bioline kits; Pf/pan and $P f$ specific kit) and PCR in the detection and speciation of Plasmodium species in children and pregnant women in Bangolan village.

\section{Materials and Methods}

\subsection{Study Area}

The study was conducted in BBIHC (the Bangolan Baptist Integrated Health Centre) found at latitude $5.50^{\circ}$ and $5.55^{\circ} \mathrm{N}$, and longitude $10.37^{\circ}$ and $10.40^{\circ} \mathrm{E}$. Bangolan village is made up of 12,000 inhabitants in 11 neighbourhoods. The village has two raining seasons- a major rainy season from March/April to August/September, and a minor one from October to November. It has an annual rainfall of $1,734.25 \mathrm{~mm}$ per annum, a daily temperature range of $27-34{ }^{\circ} \mathrm{C}$. The vegetation is guinea savannah. This village has suffered greatly from the repercussions of the Bamendjin dam constructed in 1975 and it is one of the villages that were not displaced by water. The area is perennially marshy and the people of Bangolan have embarked on rice farming and fishing for their livelihood. The abundance of marshes explains why malaria is transmitted perennially in this area.

\subsection{Study Design}

Sample collection was done between September and November 2007. A total of 70 patients who sought treatment at the BBIHC were recruited based on clinical signs and symptoms of malaria. The objective of the study was explained to participants or legal guardians who signed the informed assent or consent form prior to sample collection. The enrolled participants consisted of 16 pregnant women aged 180-420 months and 54 children aged 6-168 months. A total of $200 \mu \mathrm{L}$ of blood collected from each patient by finger prick was used to prepare thick and thin blood smears, perform RDTs and blood spot on filter paper.

2.2.1 Microscopic Examination and Determination of Parasitemia

Thick films stained with $10 \%$ Giemsa were used for definitive parasites counts; 200 high power fields were screened before a slide was declared negative. The number of parasites per 200 leucocytes was recorded and converted into parasite density per $\mu \mathrm{L}$ by 
assuming an average white blood cell count of $8,000 / \mu \mathrm{L}$. The mean of 2 slide readings was performed and discrepancies greater than $10 \%$ were performed by the microscopy quality assurance expert. Meanwhile the thin film was used for Plasmodium species identification.

\subsubsection{Rapid Diagnostic Tests}

Two types of RDTs were used which are SD Bioline Malaria Ag Pf/Pan and SD Bioline Malaria Ag $P f$ test kits (Standard Diagnostic Inc.). The Malaria $\mathrm{Ag} P f / \mathrm{Pan}$ antigen rapid tests is a one step, qualitative and rapid immunochromatographic test for the differential diagnosis of $P$. falciparum histidine rich protein II (P.f HRP-II) and the other Plasmodium species (Pan, pLDH). Meanwhile the Malaria Ag Pf has one line for control and the other for P.f HRP-II. Blood $(5 \mu \mathrm{L})$ collected from patients was used to perform the tests according to the manufacturer's instructions.

2.2.3 DNA Extraction and Amplification of the 18ss rRNA Genes by Nested PCR

DNA was extracted from dried blood spot on filter papers (Protein Saver Card) by chelex boiling method. All materials and reagents were sterilized using the autoclave (SanoClav). Using a scissors sterilized by flaming, each blood spot on the filter paper was carefully excised and transferred in $1.5 \mathrm{~mL}$ microfuge tubes and $1 \mathrm{~mL}$ of $0.5 \%$ saponin in $1 \times$ PBS added. The scissors was flamed in between samples, dipped into distilled water and wiped with a clean tissue paper. The tubes were then inverted several times to completely immerge the filter paper and kept at $4{ }^{\circ} \mathrm{C}$ overnight. The following day the solution was discarded and the filter papers washed with $1 \mathrm{~mL}$ of $1 \times$ PBS and incubated at $4{ }^{\circ} \mathrm{C}$ for 15-30 min. During this period, $50 \mu \mathrm{L}$ of $20 \%$ chelex (sterile) were added into $150 \mu \mathrm{L}$ of nuclease free water in a $1.5 \mathrm{~mL}$ microfuge tube and place on a heat block (Bioblock) set at $100{ }^{\circ} \mathrm{C}$. After incubation, the filter papers were transferred into $1.5 \mathrm{~mL}$ eppendorf tubes containing hot $20 \%$ chelex-100 and nuclease free water. During the incubation period of 10 min each tube was vortex twice, centrifuged at 13,200 rpm for $3 \mathrm{~min}$ and the supernatant transferred into a fresh tube. Centrifugation was repeated and the final supernatant (DNA) transferred into a fresh tube and stored at $-20{ }^{\circ} \mathrm{C}$ for PCR analysis. DNA from previously confirmed positive samples was extracted alongside to serve as positive control. The amplification of the18ss rRNA gene was carried out following the method in Ref. [10] with a T3 thermal cycler (Biometra, Germany). Primers used were obtained from MR4, USA. The first round was carried out in a $20 \mu \mathrm{L}$ reaction mixture consisting of $12.75 \mu \mathrm{L}$, nuclease free water, $2.5 \mu \mathrm{L}$ of $10 \times$ Thermopol buffer (New England Biolabs, USA), $0.5 \mu \mathrm{L}$ of $10 \mathrm{mM}$ dNTPs, $1 \mu \mathrm{L}$ of 2.5 $\mu \mathrm{M}$ of each primer (rPLU5/rPLU6), $0.25 \mu \mathrm{L}$ of 5 $\mathrm{U} / \mu \mathrm{L}$ Taq DNA polymerase (New England Biolabs) and $2 \mu \mathrm{L}$ of template DNA. Amplification conditions were: $95^{\circ} \mathrm{C}$ for $15 \mathrm{~min}, 26$ cycles of $58^{\circ} \mathrm{C}$ for $2 \mathrm{~min}$, $72{ }^{\circ} \mathrm{C}$ for $5 \mathrm{~min}$, and $94{ }^{\circ} \mathrm{C}$ for $1 \mathrm{~min}$, followed by $58{ }^{\circ} \mathrm{C}$ for $2 \mathrm{~min}$ and $72{ }^{\circ} \mathrm{C}$ for $5 \mathrm{~min}$. The second round of species-specific amplification was carried out in three separate tubes each containing a single pair of primers of $\mathrm{rFAL} 1 / \mathrm{rFAL} 2$, rOVAL1/rOVAL2 and rMAL1/rMAL2. The $20 \mu \mathrm{L}$ reaction mix contained $13.75 \mu \mathrm{L}$ nuclease free water, $2.5 \mu \mathrm{L}$ of $10 \times$ Thermopol buffer, $0.5 \mu \mathrm{L}$ of $10 \mathrm{mM}$ dNTPs, $1 \mu \mathrm{L}$ of each primer $2.5 \mu \mathrm{M}, 0.25 \mu \mathrm{L}$ of Taq DNA polymerase (5 $\mathrm{U} / \mu \mathrm{L})$, and $1 \mu \mathrm{L}$ of the amplicon DNA. The cycling parameters were: $95^{\circ} \mathrm{C}$ for $15 \mathrm{~min}, 30$ cycles of $58^{\circ} \mathrm{C}$ for $2 \mathrm{~min}, 72{ }^{\circ} \mathrm{C}$ for $5 \mathrm{~min}$, and $94{ }^{\circ} \mathrm{C}$ for $1 \mathrm{~min}$, followed by $58{ }^{\circ} \mathrm{C}$ for $2 \mathrm{~min}$ and $72{ }^{\circ} \mathrm{C}$ for $5 \mathrm{~min}$. Electrophoresis of PCR products was carried out on $1.5 \%$ agarose gel stained with ethidium bromide alongside molecular weight markers, positive and negative controls. Products were visualized with high performance UV transilluminator, scored and photographed using a digital camera.

\subsection{Data Analysis}

Sensitivity, specificity, negative predictive value 

Speciation in Bangolan, North West Region of Cameroon

(NPV), positive predictive value (PPV), percentage agreement (PA), and Cohen's Kappa coefficient (K) with their $95 \%$ confidence interval (CI) were determined using Open Epi version 2.2. Kappa was determined according to Altman. [11]. Kappa value express agreement beyond chance and a $\mathrm{K}$ value of 0.21-0.60 is moderate, $\mathrm{K}$ value of 0.61-0.80 a good and $\mathrm{K}>0.80$ an almost perfect agreement beyond chance. Other variables measured included true negative (TN), true positive (TP), false positive (FP), and false negative (FN). Sensitivity was calculated as $\mathrm{TP} /(\mathrm{TP}+\mathrm{FN})$, specificity as $\mathrm{TN} /(\mathrm{TN}+\mathrm{FP})$, NPV as $\mathrm{TN} /(\mathrm{TN}+\mathrm{FN}), \mathrm{PPV}$ as $\mathrm{TP} /(\mathrm{TP}+\mathrm{FP})$ and $\mathrm{PA}$ as (TP $+\mathrm{TN}) /$ sample size.

\subsection{Ethical Clearance}

Ethical clearance for the study was obtained from the Institutional Review Board of Cameroon Baptist Convention Health Board and the National Ethics Committee of the Ministry of Public Health, Cameroon.

\section{Results}

Among the 70 participants enrolled in this study, 54/70 (77.14\%) were children while 16/70 (22.85\%) were pregnant women. The mean age and weight for the children was 42 months and $14.17 \mathrm{~kg}$ respectively while that for the women were 271 months and $67.63 \mathrm{~kg}$, respectively (Table 1).

\subsection{Comparison of PCR with Microscopy}

Out of the 70 patients tested by microscopy, $61 / 70$ $(87.14 \%)$ tested positive for malaria parasites. Though microscopy failed to speciate the causative Plasmodium species, one case identified as $P$. falciparum/P. ovale/P. malariae mixed infections was confirmed by $\mathrm{PCR}$ as $P$. falciparum $/ P$. malariae mixed infections. Among the microscopically unidentified Plasmodium spp. (60/70) PCR identified 48 of the samples as $P$. falciparum/malariae mixed infections, 08 as $P$. falciparum, P. malariae and $P$. ovale mixed infections and 03 as $P$. malariae single infections (Table 2). A comparative evaluation of PCR with parasitemia indicated $33.33 \%$ detection by PCR when parasitemia was negative (Table 3).

\subsection{Comparison of PCR with Rapid Diagnostic Tests}

Of the 45 Pf/Pan kits tested, 39 were positive, PCR confirmed 30 samples and 7 samples as $P f / P m$ and $\mathrm{P} / \mathrm{Pm} / \mathrm{Po}$ mixed infections respectively. Out of the 25 $P f$ specific kit tested, PCR confirmed 18 samples and 1 sample as $P f / P m$ and $P f / P m / P o$ mixed infections

Table 1 Baseline characteristics of the study population.

\begin{tabular}{lll}
\hline & Children & Pregnant Women \\
\hline Number of participants & 54 & 16 \\
Mean age (months $\pm \mathrm{SD})$ & $41.74( \pm 38.38)$ & $270.88( \pm 48.16)$ \\
Mean weight $(\mathrm{kg} \pm \mathrm{SD})$ & $14.17( \pm 8.97)$ & $67.63( \pm 13.37)$ \\
Temperature $\left({ }^{\circ} \mathrm{C}\right)$ range & $36.0-40.2$ & $35.2-38.2$ \\
Geo-mean parasitemia $(\mathrm{SD})$ & $37,619.06( \pm 33,599.04)$ & $7,512.5( \pm 12,446.11)$ \\
\hline
\end{tabular}

SD: standard deviation.

Table 2 Comparison of PCR with microscopy for the identification of Plasmodium species in Bangolan.

\begin{tabular}{lllcc}
\hline PCR & \multicolumn{3}{c}{ Microscopy } \\
\cline { 2 - 5 } & Negative & Pf/Po/Pm & Plasmodium & Total \\
\hline Negative & 6 & 0 & 1 & $7(10 \%)$ \\
$(P f / P o / P m) \dagger$ & 0 & 0 & 8 & $8(11.43 \%)$ \\
$P f / P m$ & 2 & 1 & 48 & $51(72.86 \%)$ \\
$P m$ & 1 & 0 & 3 & $4(5.71 \%)$ \\
Total & $9(12.86 \%)$ & $1(1.43 \%)$ & $60(85.71 \%)$ & 70 \\
\hline
\end{tabular}

Pf, P. falciparum; Pm, P. malariae; Po, P. ovale. $\dagger$ : means microscopy failed to speciate the causative Plasmodium species. 

Speciation in Bangolan, North West Region of Cameroon

Table 3 Comparative evaluation of PCR with parasitemia in children and pregnant women in Bangolan.

\begin{tabular}{|c|c|c|c|c|c|c|c|}
\hline (parasites $/ \mu \mathrm{L}$ blood) & $\mathrm{Pf} / \mathrm{Pm}$ & $\mathrm{P} / \mathrm{Pm} / \mathrm{Po}$ & $\mathrm{Pm}$ & Negative & Total & \multicolumn{2}{|c|}{ Sensitivity, $\%$ Confidence level $=95 \%$} \\
\hline $500-1000$ & 3 & 0 & 1 & 0 & 4 & 100 & $51.01-100$ \\
\hline $1001-5000$ & 7 & 0 & 0 & 1 & 8 & 87.5 & $52.91-97.76$ \\
\hline $5001-10000$ & 4 & 1 & 1 & 0 & 6 & 100 & $60.97-100$ \\
\hline $10001-20000$ & 8 & 0 & 0 & 0 & 8 & 100 & $67.56-100$ \\
\hline 20001-30000 & 9 & 0 & 1 & 0 & 10 & 100 & $72.25-100$ \\
\hline $30001-40000$ & 4 & 1 & 0 & 0 & 5 & 100 & $56.55-100$ \\
\hline $40001-50000$ & 2 & 0 & 0 & 0 & 2 & 100 & $34.24-100$ \\
\hline $50001-60000$ & 3 & 2 & 0 & 0 & 5 & 100 & $56.55-100$ \\
\hline $60001-70000$ & 2 & 2 & 0 & 0 & 4 & 100 & $51.01-100$ \\
\hline $70001-80000$ & 1 & 1 & 0 & 0 & 2 & 100 & $34.24-100$ \\
\hline $80001-90000$ & 3 & 0 & 0 & 0 & 3 & 100 & $48.85-100$ \\
\hline $90001-100000$ & 2 & 1 & 0 & 0 & 3 & 100 & $48.85-100$ \\
\hline$>100000$ & 1 & 0 & 0 & 0 & 1 & 100 & $20.66-100$ \\
\hline negative & 2 & 0 & 1 & 6 & 9 & 33.33 & $12.06-64.58$ \\
\hline Total & 51 & 8 & 4 & 7 & 70 & 90 & $80.77-95.07$ \\
\hline
\end{tabular}

Table 4 Comparison of PCR and RDTs in the speciation of Plasmodium species in children and pregnant women in Bangolan.

\begin{tabular}{llllll}
\hline PCR & \multicolumn{5}{c}{ RDTs } \\
\cline { 2 - 6 } & Pf/Pan (3) & $P f(2)$ & $P f *$ & Pf/Pan* & Total \\
\hline -VE & 1 & 0 & 3 & 3 & $7(10 \%)$ \\
Pf/Pm & 30 & 18 & 0 & 3 & $51(72.86 \%)$ \\
Pf/Pm/Po & 7 & 1 & 0 & 0 & $8(11.43 \%)$ \\
Pm & 1 & 2 & 1 & 0 & $4(5.71 \%)$ \\
Total & $39(55.71 \%)$ & $21(30 \%)$ & $4(5.71 \%)$ & $6(8.57 \%)$ & 70 \\
\hline
\end{tabular}

Pf* and Pf/Pan* denotes invalid results; $P f(2)$ denotes presence of two bands (Plasmodium spp and control line); Pf/Pan (3) denotes the presence of three bands ( $P f$, pan species and control line).

Table 5 The sensitivity and specificity of RDTs kits compare to PCR in the diagnosis of malaria in the study population.

\begin{tabular}{lllllll}
\hline \multirow{2}{*}{ RDTs } & \multicolumn{5}{c}{ Nested PCR } \\
\cline { 2 - 7 } & Sensitivity, \% & Specificity, \% & PPV $^{*} \%$ & NPV**, \% & Accuracy, \% & Kappa \\
\hline \multirow{2}{*}{$P f$ kit $^{\text {a }}$} & 95.45 & 100 & 100 & 75 & 97.44 & 0.8344 \\
& $(78.2-99.19)$ & $(43.85-100)$ & $(84.54-100)$ & $(30.06-95.44)$ & $(80.46-99.29)$ & $(0.448-1.22)$ \\
\multirow{2}{*}{$P f /$ Pan $^{b}$ kit } & 92.68 & 75 & 97.44 & 50 & 91.11 & 0.552 \\
& $(80.57-97.48)$ & $(30.06-95.44)$ & $(86.82-99.55)$ & $(18.76-81.24)$ & $(79.27-96.49)$ & $(0.2675-0.837)$ \\
\hline
\end{tabular}

*PPV: positive predictive value; **NPV: negative predictive value; CI: confidence interval $95 \%$;

${ }^{\mathrm{a}}$ : the limit of detection of $P f$ kit was $480 / \mu \mathrm{L}$ in children and $520 / \mu \mathrm{L}$ in women; ${ }^{\text {b }}$ : the limit of detection of $P f / P a n$ kit was $400 / \mu \mathrm{L}$ in children and 2,560 $\mu \mathrm{L}$ in women; $95 \%$ confidence intervals are indicated in parentheses.

respectively (Table 4). Three samples diagnosed by the $P f / P a n$ and $P f$ specific kit as $P$. falciparum were identified as $P$. malariae single infection by PCR (Table 4). The detection range for parasitemia was from 400-99,600 parasites/ $\mu \mathrm{L}$ for Pf/Pan kits and from $480-149,600$ parasites/ $\mu \mathrm{L}$ for $P f$ specific kit in the children population. Meanwhile, for the 16 pregnant women the detection range for parasitemia was $520-38,400$ parasites $/ \mu \mathrm{L}$ for $P f \quad$ kit and
2560-28,320 parasites/ $\mu \mathrm{L}$ with $P f /$ Pan specific kit. Amongst the three methods, PCR was observed to be the most sensitive and specific method (Tables 5 and 6). The concordance between PCR and Pf specific kit was higher than PCR and Pf/pan kits. The Kappa value between PCR and RDTs was observed to be higher than that between PCR and microscopy in the pregnant women population (Table 6).

$P f / P a n$ and $P f$ results were invalid tests because in 
Table 6 Sensitivity and specificity of nested PCR, microscopy and RDTs in the diagnosis of malaria in the study population.

\begin{tabular}{lllllll}
\hline & \multicolumn{5}{c}{ Nested PCR } \\
\cline { 2 - 7 } & Sensitivity, $\%$ & Specificity, $\%$ & PPV, $\%$ & NPV, $\%$ & Accuracy, \% & Kappa \\
\hline \multirow{2}{*}{ MIC } & 100 & 66.67 & 70 & 100 & 81.2 & 0.64 \\
& $(64.57-100)$ & $(35.42-87.94)$ & $(39.68-89.22)$ & $(60.97-100)$ & $(56.99-93.41)$ & $(0.18-1.10)$ \\
\multirow{2}{*}{ RDTs } & 100 & 75 & 80 & 100 & 87.5 & 0.75 \\
& $(64.57-100)$ & $(40.93-92.85)$ & $(49.02-94.33)$ & $(60.97-100)$ & $(63.98-96.5)$ & $(0.28-1.22)$ \\
\hline
\end{tabular}

MIC: microscopy; RDTs: Rapid diagnostic tests; PPV: positive predictive value; NPV: negative predictive value; $95 \%$ confidence intervals are indicated in parentheses.

the course of reaction no control line was observed in the test strip. $P f(2)$ : kit specific for Plasmodium falciparum and (2) denotes the presence of two bands (one for $P f$ and the other for the control line). Pf/Pan (3): kit specific for P. falciparum and pan species ( $P$. vivax, P. ovale, P. malariae) and (3) denote the presence of three bands (one for Pf, one for pan species and one for the control line).

\section{Discussion}

Microscopy still remains the method of choice in the diagnosis of malaria in endemic areas because it is an inexpensive, can serve for more than just plasmodium diagnosis and may also provide information species and the state of anaemia. However, this method can sometimes be burdensome, light failures and reagent inadequacies. In addition, the identification of parasite species, especially in the case of low level of parasitemia and a mixed parasite infection can be tricky especially to the untrained eye $[12,13]$. In this study the sensitivities and specificity of microscopy, PCR and RDTs in the detection and identification of Plasmodium species in children less than 5 years and pregnant women were compared. The nested PCR technique used in this study permitted the identification of Plasmodium falciparum, Plasmodium ovale, and Plasmodium malaria as the three malaria parasites currently found in Bangolan. The parasites as identified by PCR were classified as double (Pf/Pm), triple $(\mathrm{P} / \mathrm{Po} / \mathrm{Pm})$ and single $(\mathrm{Pm})$ infections. The prevalence of mixed infections is often biased by microscopy especially with $P$. falciparum that overshadows the other species by reason of low parasitemia. This study confirms previously reports that PCR is more sensitive and specific in the detection of Plasmodium species and can easily differentiate between mix infections. Thin film microscopic differentiation between $P f$ and $P m$ has been reported to be difficult [14] and although $P m$ is a mild infection, it could greatly affect the dynamic of $P f$ manifestation through non-specific and cross-specific immune response [15] that can cause a chronic nephrotic syndrome. Once established, this syndrome does not respond to treatment and carries a high rate of mortality [16]. Moreover, $P f / P m$ are known to co-infect together in endemic areas [17]. In this study, the most prevalent mixed infection was observed to be $\mathrm{Pf} / \mathrm{Pm}$ (77.86\%) compared to $\mathrm{Pf} / \mathrm{Pm} / \mathrm{Po}(11.43 \%)$ and no single $P$. falciparum infection was observed. Some of the factors that have been reported to influence microscopic results are the experience of the microscopist, appropriate staining and the time in reading the slides. The inability of microscopy to clearly speciate the Plasmodium species in this study might have been influenced by the above factors. Previous studies have reported the sensitivity of PCR in very low parasitemia $[11,18,19]$. A similar trend was observed where $18.75 \%$ of aparasitemic pregnant women detected by microcopy were identified by PCR. Hence, though PCR is not a prompt diagnostic method and is not easily affordable, may help in managing treatment of malaria in pregnant women knowing that treatment on the sole basis of microscopy can lead to under-treatment and sometimes over treatment. Gravidity has been reported to influence the occurrence of $P$. falciparum and parasites densities but 
not the prevalence of $P$. ovale and P. malariae [20]. Thus, this may explain the reason why PCR failed to detect $P$. falciparum in some samples observed to be $P$. falciparum positive by RDTs kits. A study carried out in Burkina Faso by Mockenhaupt et al. who [21] reported that MAKROmed RDT could detect parasites at a submicroscopic level. Similarly in this study two samples that were negative by microcopy were observed to be positive by RDTs and PCR. Moreover, the sensitivity of microscopy, RDTs and PCR were at $87.14 \%, 85.71 \%$ and $90 \%$ respectively which agrees with previously reported trend [22]. Pregnant women are the most at risk in this case where $P$. falciparum sequesters in the placenta leading to drastic effects especially in primigravidae. The sensitivity of PCR at low parasitemia was reported in Refs. [11, 19], the authors found that PCR can be effective at 6 parasites $/ \mu \mathrm{L}$ or even less. In this study, we obtained $18.75 \%(3 / 16)$ of aparasitemic women by PCR, while Singer et al. [20] reported $46 \%$ (164/358), the difference could be due to the small sample. Nevertheless, in this context PCR appears to be limited because it cannot evaluate the parasite density, which is of crucial importance in following the epidemiology of parasites load in a given setting [23]. In the present study, it was also observed that the Cohen's Kappa agreement between PCR and RDTs was higher ( $\mathrm{K}=$ $0.75, \mathrm{CI}=0.28-1.22)$ than that between PCR and microscopy $(\mathrm{K}=0.64, \mathrm{CI}=0.18-1.10)$. Moreover, Boonma et al. [24] and Imwong et al. [25] had reported a decrease in the sensitivity of PCR when compared to microscopy as gold standard. The findings from this study therefore indicate that RDTs used could be a good tool for diagnosis after PCR. Although PCR is of great importance in distinguishing malarial species it appears to be limited when sequence variations occur within genomic DNA. In this study, it was observed that primers used to detect $P$. malariae infection were found to cross-react with $P$. ovale genomic DNA in 9/70 of the samples tested. Kawamoto et al. [26] reported a similar observation in their study on spurious amplification of $P$. vivax small subunit RNA gene using the primers for $P$. knowlesi. This situation could probably be due to sequence variations in $P$. ovale 18SS rRNA gene as reported by Basco and Tahar [27]. Although cross-reactivity of P. malariae primers with $P$. ovale might be reduced by further optimization of the amplification conditions it is probable that a new set of primers truly specific for $P$. malariae would be preferable.

In summary, although the cost effective microscopy still remains the gold standard for parasitological diagnosis of malaria in field settings, RDTs are becoming popular for routine diagnosis of malaria in vulnerable groups and PCR still remains the most sensitive and specific method. Malaria infection in Bangolan is mostly due to mix infection predominantly $P$. falciparum/P. malariae and this could influence treatment response. With the current plans of the National Malaria Program to introduce RDTs for malaria diagnosis at the point of care in all health facilities nationwide, these findings provide useful information for the type of RDT that could be used for effective case management of malaria in this area.

\section{Acknowledgments}

The research leading to these results has received funding through Prof. Mbacham from IAEA RAF/ 6036, the European Union Seventh Framework Programme (FP7/2007-2013) under grant agreement $\mathrm{n}^{\circ}$ 242095-Pr.\#31- EVIMalaR, the University of Yaounde I, Cameroon - Pr. \# UYI/FS/64.89/wfm and and Fobang Foundation $-\mathrm{AD} / \mathrm{FF} / 03 / 08$. The authors are grateful to the staff of Bangolan Baptist Integrated Health Center, pregnant women and children in Bangolan village who participated in the study.

\section{References}

[1] WHO, Malaria case management, operational manual, Global Malaria Programmes, 2009.

[2] J. Sachs, P. Maloney, The economic and social burden of malaria, Nature 415 (7) (2002) 680-685.

[3] R.W. Steketee, B.L. Nahlen, M.E. Parise, C. Menendez, 

Speciation in Bangolan, North West Region of Cameroon

The burden of malaria in pregnancy in malaria-endemic areas, American Journal of Tropical Medicine and Hygiene 64 (2) (2001) 28-35.

[4] H.M. Gilles, Diagnostic methods in malaria, in: H.M. Gilles, D.A. Warrell (Eds.), Bruce-Chwatt's Essential Mariology, 3rd ed., Edward Arnold, 1993, pp. 78-95.

[5] WHO, The Use of Malaria Rapid Diagnostic Tests, 2nd ed., WHO-TDR/WHO-WPRO, Geneva, 2006.

[6] C.K. Murray, D. Bell, R.A. Gasser, C. Wongsrichanalai, Rapid diagnostic testing for malaria, Tropical Medicine and International Health 8 (2003) 876-883.

[7] F. Kawamoto, H. Miyake, O. Kaneko, M. Kimura, T.D. Nguyen, Q. Lui, et al., Sequence variation in the $18 \mathrm{~S}$ rRNA gene, a target for PCR-based malaria diagnosis in Plasmodium ovale from southern Vietnam, Journal of Clinical. Microbiology 34 (1996) 2287-2289.

[8] G. Snounou, S. Viriyakosol, W. Jarra, S. Thaithong, K.N. Brown, Identification of the four human malarial species in field samples by the polymerase chain reaction and detection of a high prevalence of mixed infections, Molecular and Biochemical. Parasitology 58 (1993) 283-292.

[9] P. Boonma, R.P. Christensen, R. Suwanarusk, N.R. Price, B. Russel, U. Lek-Uthai, Comparison of three molecular methods for the detection and speciation of Plasmodium vivax and Plasmodium falciparum, Malaria Journal 124 (6) (2007) 1-7.

[10] G. Snounou, S. Viriyakosol, X.P. Zhu, W. Jarra, L. Pinheiro, V.E. Rosario, et al., High sensitivity of detection of human malaria parasites by the use of nested polymerase chain reaction, Molecular and Biochemical Parasitology 61 (1993) 315-320.

[11] D.G. Altman, Practical Statistics for Medical Research, Chapman \&Hall, London, 1991.

[12] R. Knowles, R.S. White, Studies in the parasitology of malaria, Calcutta, India, Indian Medical Research Memoirs 18 (1930) 436.

[13] T.L. Richie, Interactions between malaria parasites infecting the same vertebrate host, Parasitology 96 (1988) 607-639.

[14] I. Mueller, P.A. Zimmerman, J.C. Reeder, Plasmodium malariae and Plasmodium ovale - the 'bashful' malaria parasites, Trends in Parasitology 23 (6) (2007) 278-283.

[15] D.P. Mason, F.E. McKenzie, W.H. Bossert, The blood-stage dynamics of mixed Plasmodium malariae-Plasmodium falciparum infection, Journal of Theoretical Biology 198 (1999) 549-566.

[16] S. Eiam-Ong, Malaria nephropathy, Seminars in Nephrology 23 (1) (2003) 21-33.

[17] W.E. Collins, G.M. Jeffery, Plasmodium malariae:
Parasite and disease, clinical, Microbiology Review 20 (4) (2007) 579-592.

[18] B. Singh, A. Bobogare, J. Cox-Singh, G. Snounou, M.S. Abdullah, H.A. Rahman, A genus- and species-specific nested polymerase chain reaction Malaria detection assay for epidemiologic studies, American Journal of Tropical Medicine and Hygiene 60 (4) (1999) 687-692.

[19] F.P. Mockenhaupt, B.T. Rong, T. Holger, T.A. Eggette, S. Beck, C. Gyasi-Sarpong, et al., Submicroscopic Plasmodium falciparum infection in pregnancy in Ghana, Tropical Medicine and International Health 5 (3) (2000) 167-173.

[20] L.M. Singer, R.D. Newman, A. Diarra, A.C. Moran, C.S. Huber, G. Stennies, et al., Evaluation of a malaria diagnostic test for assessing the burden of malaria during pregnancy, American Journal of Tropical Medicine and Hygiene 70 (5) (2004) 481-485.

[21] F. Mockenhaupt, G. Bedu-Addo, C.V. Gaertner, R. Boyé, K. Fricke, I. Hannibal, et al., Detection and clinical manifestation of placental malaria in Southern Ghana, Malaria Journal 119 (5) (2006) 1-10.

[22] P.F. Mens, G.J. Schoone, P.A. Kager, D.F.H. SchalligHenk, Detection and identification of human Plasmodium species with real-time quantitative nucleic acid sequence-based amplification, Malaria Journal 5 (80) (2006) 1-6.

[23] R.E. Coleman, J. Sattabongkot, S. Promstaporm, N. Maneechai, B. Tippayachai, A. Kengluecha, Comparison of PCR and microscopy for the detection of asymptomatic malaria in a Plasmodium falciparun/vivax endemic area in Thailand, Malaria Journal 5 (21) (2006) $1-22$.

[24] P. Boonma, R.P. Christensen, R. Suwanarusk, N.R. Price, B. Russel, U. Lek-Uthai, Comparison of three molecular methods for the detection and speciation of Plasmodium vivax and Plasmodium falciparum, Malaria Journal 124 (6) (2007) 1-7.

[25] M. Imwong, N. Tanomsing, S. Pukrittayakamee, N.P.J. Day, N.J. White, G. Snounou, Spurious amplification of Plasmodium vivax small subunit RNA gene using the primers currently used to detect Plasmodium knowlesi, Journal of Clinical Microbiology 47 (2009) 4173-4175.

[26] F. Kawamoto, H. Miyake, O. Kaneko, M. Kimura, T.D. Nguyen, N. Dung, et al., Sequence variation in the $18 \mathrm{~S}$ rRNA gene, a target for PCR-based Malria diagnosis, in Plasmodium ovale from Southern Vietnam, Journal of Clinical Microbiology 34 (9) (1996) 2287-2289.

[27] L.K. Basco, R. Tahar, Detection of Plasmodium ovale malaria parasites by species-specific of 18S rRNA gene Amplification, Molecular and Cellular Probes 11 (1997) 389-395. 